\title{
Reverse Time Migration: A Seismic Imaging Technique Applied to Synthetic Ultrasonic Data
}

\author{
Sabine Müller, ${ }^{1}$ Ernst Niederleithinger, ${ }^{1}$ and Thomas Bohlen ${ }^{2}$ \\ ${ }^{1}$ Division 8.2 of Non-destructive Damage Assessment and Environmental Measurement Methods, \\ BAM Federal Institute for Materials Research and Testing, Unter den Eichen 87, 12205 Berlin, Germany \\ ${ }^{2}$ Geophysical Institute, KIT Karlsruhe Institute of Technology, Hertzstr. 16, 76187 Karlsruhe, Germany
}

Correspondence should be addressed to Sabine Müller, sabine.mueller@bam.de

Received 6 February 2012; Revised 24 May 2012; Accepted 25 May 2012

Academic Editor: Joerg Schleicher

Copyright (c) 2012 Sabine Müller et al. This is an open access article distributed under the Creative Commons Attribution License, which permits unrestricted use, distribution, and reproduction in any medium, provided the original work is properly cited.

\begin{abstract}
Ultrasonic echo testing is a more and more frequently used technique in civil engineering to investigate concrete building elements, to measure thickness as well as to locate and characterise built-in components or inhomogeneities. Currently the Synthetic Aperture Focusing Technique (SAFT), which is closely related to Kirchhoff migration, is used in most cases for imaging. However, this method is known to have difficulties to image steeply dipping interfaces as well as lower boundaries of tubes, voids or similar objects. We have transferred a processing technique from geophysics, the Reverse Time Migration (RTM) method, to improve the imaging of complicated geometries. By using the information from wide angle reflections as well as from multiple events there are fewer limitations compared to SAFT. As a drawback the required computing power is significantly higher compared to the techniques currently used. Synthetic experiments have been performed on polyamide and concrete specimens to show the improvements compared to SAFT. We have been able to image vertical interfaces of step-like structures as well as the lower boundaries of circular objects. It has been shown that RTM is a step forward for ultrasonic testing in civil engineering.
\end{abstract}

\section{Introduction}

Quality assurance and damage assessment of concrete structures as buildings, bridges, and dams are major tasks in civil engineering. On one side construction drawings may be missing or are considered to be unreliable, which is a drawback for maintenance and rehabilitation measures. On the other side parts of the public infrastructure, namely, bridges, suffer from aging, increased traffic, and increasing individual truck weight. In addition nondestructive inspection methods are required to provide a reliable quality assurance for new, repaired, or rebuilt structures. Since about twenty years, ultrasonic techniques are more and more frequently used to map the interior of structures, for example, to locate layers, voids, objects, or other features. The localisation and characterisation of tendon ducts is of major importance. These ducts are used to apply external forces to the concrete via prestressed steel wires to enhance its tension resistance. After prestretching these wires, the duct is grouted with mortar in order to couple the wires and the concrete as well as to avoid corrosion. Remaining air voids are a problem for the durability of the structure. In many cases the first step in an assessment is to locate the tendon ducts, because quite often they are not placed in accordance with the design plans.

Until 20 years ago, ultrasonic testing in civil engineering was limited to relatively simple methods as time of flight transmission measurements to assess concrete quality. Meanwhile echo measurements are a well-established nondestructive testing method in civil engineering. Recently, the introduction of new transmitters and receivers, array techniques, and data processing methods has led to the development of imaging applications in research and practice. The data acquisition geometries as well as wave phenomena are similar to the ones in seismic techniques, but on a different scale. Imaging is mainly done using SAFT (synthetic aperture focusing technique), which is closely related to Kirchhoff migration. Thereby it suffers from the same limitations, for example, problems with imaging of vertical or steeply dipping interfaces. Measurements are mainly done using a zero offset geometry. That means that receiver and 

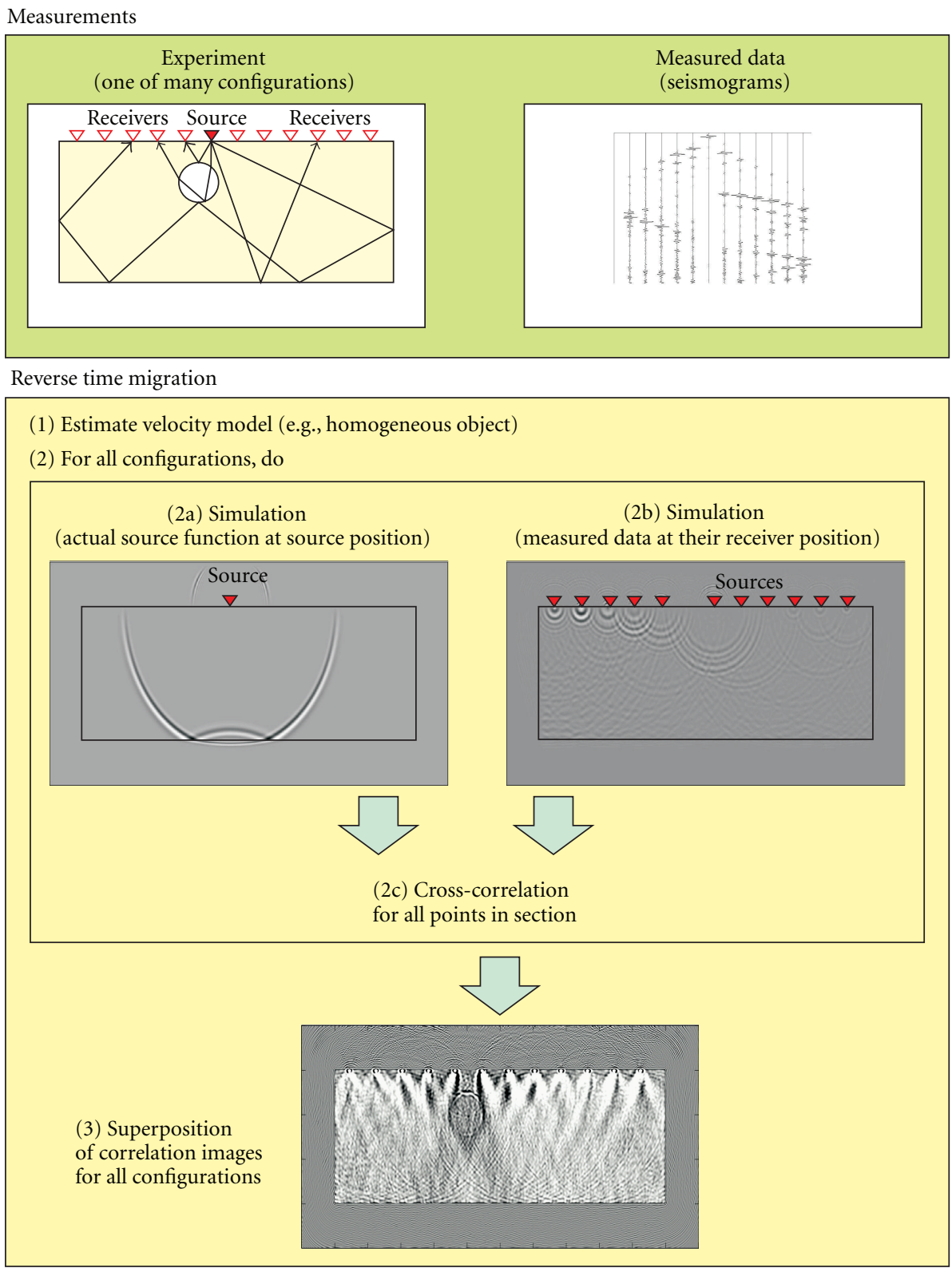

Figure 1: Principle of reverse time migration.

transmitter have a constant distance of some centimetres. By this reason only the top of, for example, tendon ducts can be imaged. The duct also shadows the back wall and any other structural features behind it. Details on SAFT algorithms applied to measurements on concrete have been published by Mayer et al. [1] and Schickert et al. [2]. Krause et al. [3] have compiled the state of the art on imaging grouting faults in tendon ducts. An extension of the method using phase analysis of the measured signal reflections was published by Krause et al. [4]. In exploration seismics several methods have been introduced to image complex structures including steeply dipping interfaces. A promising technique called reverse time migration (RTM) was originally introduced by Baysal et al. [5], Loewenthal and Mufti [6], and McMechan [7] in 1983. However, due to the required computing power, it was not until after 2000 that RTM became a practicable method. Some examples of recent applications of RTM in hydrocarbon exploration are shown by Farmer et al. [8]. Other authors have experimented successfully with the application of RTM in structural health monitoring of composites, for example, crack detection (Zhou et al. [9], Wang and Yuan [10]).

In this paper we show the potential of RTM for ultrasonic testing in civil engineering and the advantages compared to SAFT using synthetic models.

\section{Reverse Time Migration}

The basic idea of RTM is a three-step procedure of (a) forward modelling of a wave field through an appropriate 


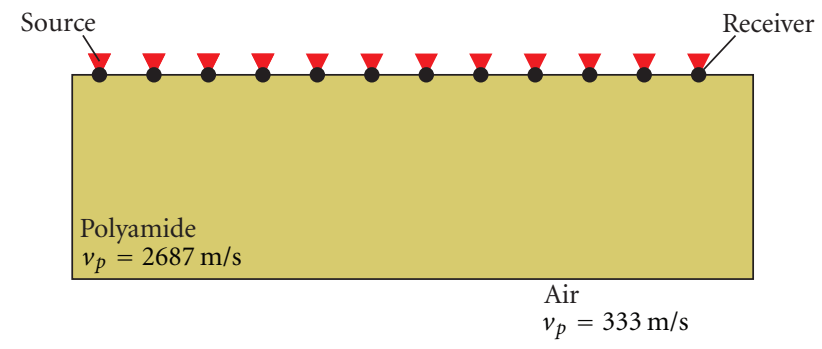

FIGURE 2: Homogeneous velocity model for migration.

velocity model as well as (b) back propagation of the measured data through the very same model and (c) superposition of both using an imaging condition. The complete wave equation is used for the simulation. There are no dip restrictions for imaging because the entire wave field including multiple reflections is used. Thus even vertical boundaries or lower edges of objects can be imaged.

The RTM algorithm used in this paper is based on a finite difference modelling code by Bohlen [11]. Currently we are using the two-dimensional acoustic wave equation. Some improvements for full waveform inversion, acoustic modelling with perfectly matched layers as boundary condition, and shot parallelisation were done by Kurzmann et al. [12]. The code can be applied to different tasks from highfrequency ultrasonic measurements to exploration seismics. It provides a set of parameters to be adapted to the specific problem. For example, arbitrary source functions can be chosen to mimic ultrasonic transducers or explosive sources. In our examples we have used a Ricker wavelet as source signal and perfectly matched layers as boundary condition.

Figure 1 shows the principle of RTM for a 2D case with coordinates $x$ (horizontal) and $z$ (vertical). In an experiment with several sources and receivers measurement data are acquired. The recording time must be sufficiently long to include multiple reflections. A velocity model has to be chosen for the imaging process. A homogeneous or smoothed model is used in most cases. For all shot point configurations two simulations are performed using the very same model: (a) forward simulation using an appropriate source function at the shot point to calculate the wave field $S_{s}(t, z, x)$ and (b) reverse modelling using the reversed measured data as a source function at the corresponding receiver points to calculate a second wave field $R_{s}(t, z, x)$. After the simulation an imaging condition is applied. In our case we have used cross-correlation which is normalised by the square of the source illumination strength. The correlation images of all shots are summed up to get the image $I(z, x)[13]$ :

$$
I(z, x)=\sum_{s} \frac{\sum_{t} S_{s}(t, z, x) R_{s}(t, z, x)}{\sum_{t} S_{s}^{2}(t, z, x)} .
$$

\section{Simulation of Typical Problems in Nondestructive Testing}

We have tested the RTM with synthetic data, which we had generated with the forward modelling part of the RTM code. We created several synthetic models to study different tasks in nondestructive testing. The first models represent polyamide specimen, a homogeneous, diffraction-free material. In a second step we have simulated a concrete-like structure with multiple scatterers.

We have chosen four polyamide models to show the capabilities of the algorithm: a step, two steps, an air inclusion, and a step with an air inclusion. The models consist of 1200 by 500 grid points with a distance between grid points of $d h=0.001 \mathrm{~m}$. The specimens have a maximum size of $1 \mathrm{~m}$ by $0.30 \mathrm{~m}$ and compressional wave velocity of $v_{p}=$ $2687 \mathrm{~m} / \mathrm{s}$. The specimens are surrounded by an $0.10 \mathrm{~m}$ width air layer with a velocity of $v_{p}=333 \mathrm{~m} / \mathrm{s}$. On the top surface of the polyamide specimen we positioned the 12 sources and coincidental receivers with a distance of $x=0.08 \mathrm{~m}$ between them. If a Ricker wavelet with $f=100 \mathrm{kHz}$ is inserted at every shot point, all 12 receivers are in record mode. The recording time is $t=0.6 \mathrm{~ms}$ with a time stepping of $d t=$ $0.1 \mu \mathrm{s}$. This way even multiple reflections are recorded.

For all following RTM reconstructions we have used a homogeneous, rectangular velocity model $\left(v_{p}=2687 \mathrm{~m} / \mathrm{s}\right.$ as in data generation), shown in Figure 2. This way, the algorithm has no information about the real structure, except for the outer limits and the material velocity.

3.1. Step. The first synthetic model includes a small step in the lower boundary of the polyamide specimen (Figure 3(a)). The step has a height of $z=0.10 \mathrm{~m}$. Figure $3(\mathrm{~b})$ shows the result of the RTM calculated with the homogeneous velocity model. The step is imaged in the correct position and height. The vertical border of the step is clearly visible. There are also artefacts at every multiple height of the step, which are not completely explained until now. They are probably an effect of the cross-correlation, as the velocity model used here intentionally does not include the step. So the forward wave field contains no information about the step while the back propagation (using the measured data) does. So we get a high signal in the cross-correlated image at the real step position, but also lower signals in multiple distance. The longer the recording time is, the more multiple reflections are recorded. That is why the amplitude of the artefacts increase with longer recording time. This result shows the importance to choose a proper recording time for experiments, long enough to image the entire structure, but not too long to enhance artefacts.

3.2. Two Steps. The next model consists of two steps with a height of $z=0.06 \mathrm{~m}$ for each step (Figure 4(a)). Figure 4(b) shows the migrated image generated with the homogeneous 


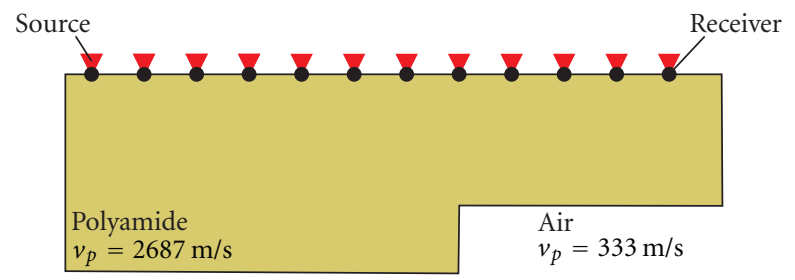

(a)

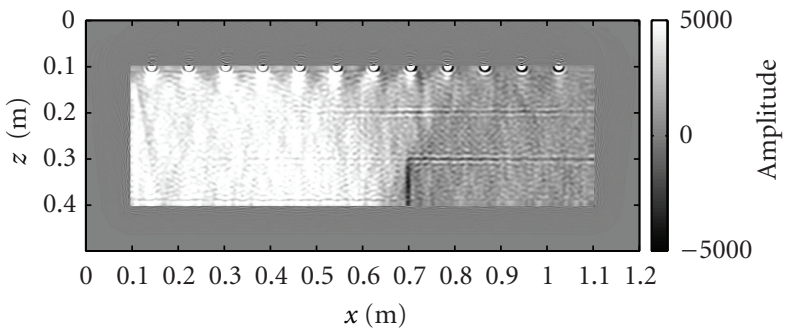

(b)

FIGURE 3: (a) Synthetic step model. (b) RTM imaging result using velocity model of Figure 2.

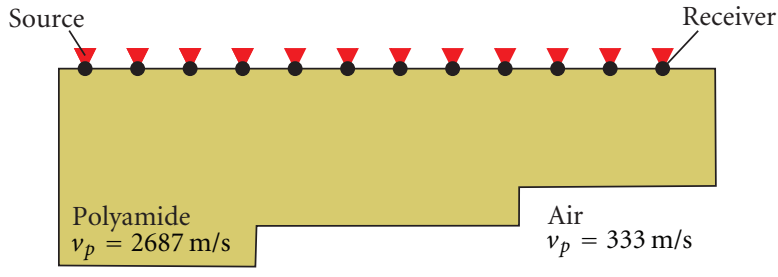

(a)

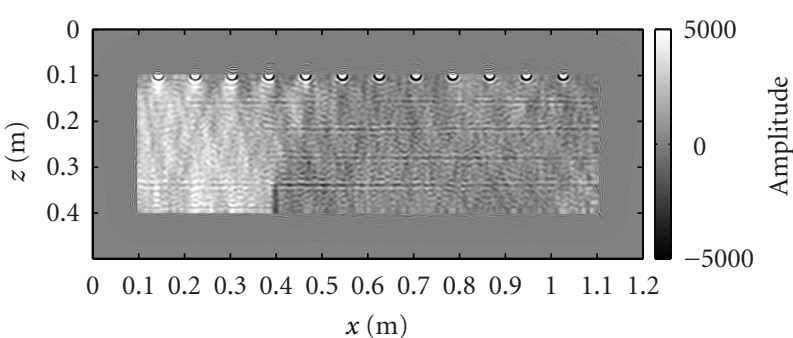

(b)

FIgURE 4: (a) Two-step model. (b) RTM imaging result using velocity model of Figure 2.

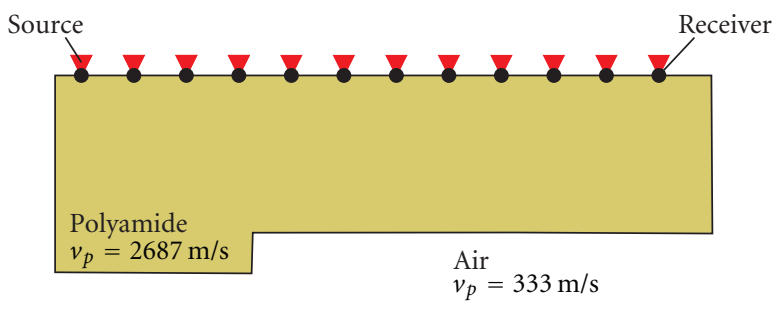

(a)

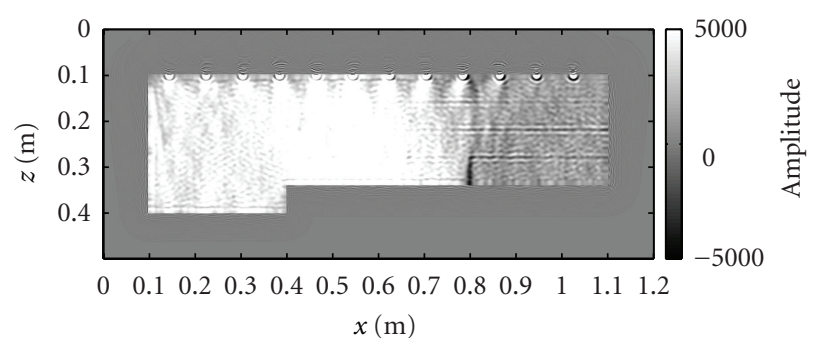

(b)

FIGURE 5: (a) Modified velocity model for migration. (b) RTM imaging result of the two steps using velocity model of (a).

rectangular model. Now only the first step is imaged at the right height and with a clear vertical border. However, the second step is missing. Only artefacts by multiple reflections are imaged. Apparently RTM fails, if the model used for reconstruction is too far from reality. Thus we have tried a kind of two-stage RTM. We used the information from the first reconstruction, the imaged step, and created a new velocity model (Figure 5(a)). Figure 5(b) shows the result of the second RTM using the improved velocity model. Now the second step is clearly visible. This example illustrates that for more complicated models a multistage RTM is required if insufficient a priori information about the structure is available.

3.3. Air Inclusion. The next model includes a circular-shaped air inclusion in polyamide. The circle has a radius of $r=$ $0.05 \mathrm{~m}$. The midpoint is at $x=0.5 \mathrm{~m}$ and $z=0.2 \mathrm{~m}$ (Figure 6(a)). Figure 6(b) shows the result of the RTM, again calculated using the homogeneous velocity model. The complete circular boundary of the inclusion is imaged.
This is an significant improvement compared to Kirchhoff migration or SAFT, which are capable of imaging the upper side of the inclusion only.

3.4. Step with Air Inclusion. The last polyamide model is a combination of the step model and a smaller air inclusion. The step has again a height of $z=0.10 \mathrm{~m}$. The midpoint of the inclusion is located at $x=0.4 \mathrm{~m}$ and $z=0.25 \mathrm{~m}$, and it has a radius of $r=0.03 \mathrm{~m}$ (Figure 7(a)). Figure 7(b) shows the migrated image. This example shows that also different defects inside a specimen can be located simultaneously. The vertical border of the step is imaged and also the boundary of the inclusion. As already seen in the first model (Section 3.1), artefacts are generated by imaging the step using a rectangular velocity model.

3.5. Scattering Model with Air Inclusion. To simulate experiments on concrete, we have designed an additional model, which includes the scattering effect of aggregates. In real concrete the size of the aggregates (e.g., gravel) is in the same 


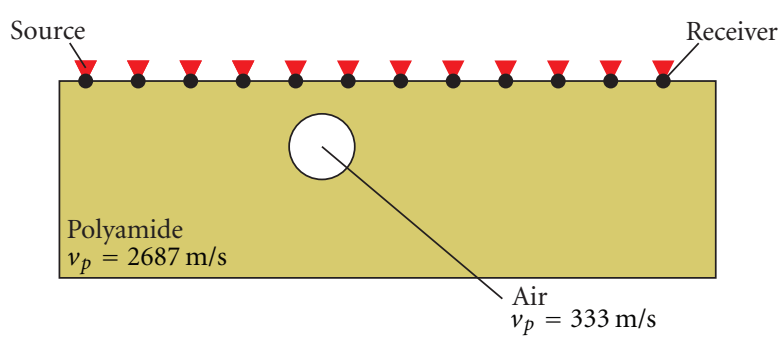

(a)

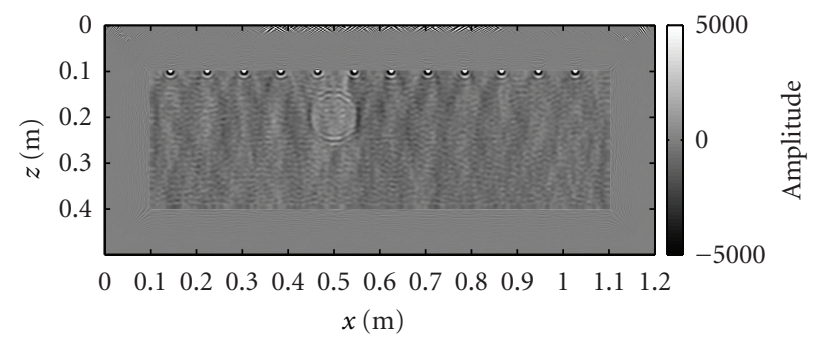

(b)

FIGURE 6: (a) Synthetic air inclusion model. (b) RTM imaging result using velocity model of Figure 2.

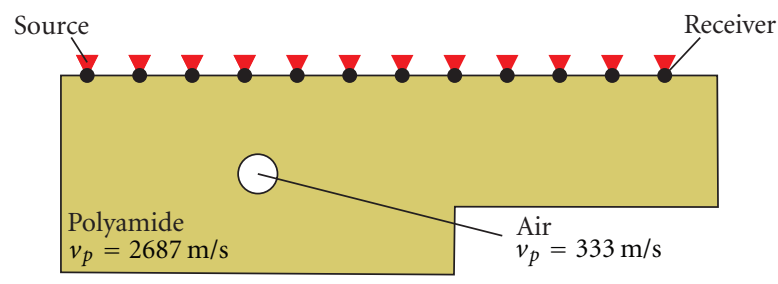

(a)

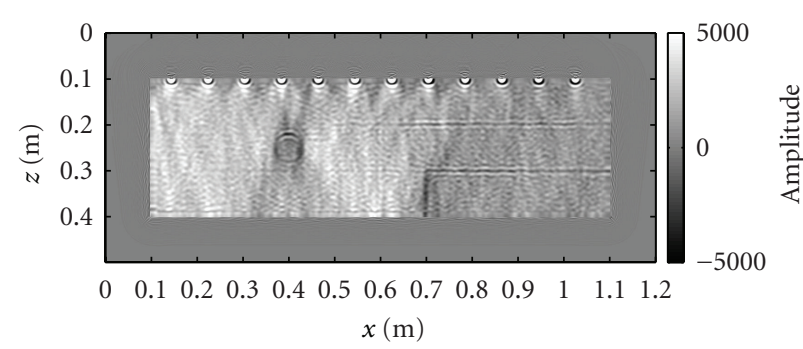

(b)

Figure 7: (a) Step with air inclusion model. (b) RTM imaging result using velocity model of Figure 2.

order of magnitude as the ultrasonic wavelength. We have arranged 300 circular scatterers in the synthetic specimen. Figure 8(a) shows the model. The model consists of $1200 \times$ 700 grid points with a distance of $d h=0.001 \mathrm{~m}$ between them. The size of the specimen is $1 \mathrm{~m}$ by $0.5 \mathrm{~m}$ surrounded by an air layer of $0.1 \mathrm{~m}$ thickness. The background velocity of the specimen is $v_{p}=4000 \mathrm{~m} / \mathrm{s}$ and of the surrounding air layer $v_{p}=333 \mathrm{~m} / \mathrm{s}$. The 300 randomly arranged scatterers have a velocity range from $3000 \mathrm{~m} / \mathrm{s}$ to $4000 \mathrm{~m} / \mathrm{s}$ and a size between $2 \mathrm{~mm}$ and $16 \mathrm{~mm}$. The midpoint of the air inclusion is located at $x=0.5 \mathrm{~m}$ and $z=0.3 \mathrm{~m}$, with a radius of $0.05 \mathrm{~m}$.

We have performed RTM with a source/receiver distribution similar to the geometry used for the polyamide models. These experiments showed no success as the scattered wave energy blurs the image. To achieve better results we have added additional receivers to average the signal. Finally we have placed 10 sources with a distance interval of $0.1 \mathrm{~m}$, and 45 receivers with a distance interval of $0.02 \mathrm{~m}$. A Ricker wavelet with $f=100 \mathrm{kHz}$ was used as source function. The signal was recorded for $0.6 \mathrm{~ms}$ with a time stepping $d t=$ $0.1 \mu \mathrm{s}$.

For the RTM we have chosen a homogeneous model again with the same background velocity as the real model, $v_{p}=4000 \mathrm{~m} / \mathrm{s}$, but without the scatterers and the inclusion (Figure 8(b)). Figure 8(c) shows the migrated image. The border of the inclusion is completely visible, but the amplitude is significantly lower than in the previous examples. Especially the left and right sides of the inclusion are imaged weakly. Increasing the record time did not improve the result. Indeed, using a longer record time more multiple reflections from the borders of the specimen are included as well as scattering noise. For this reason the signalto-noise ratio decreases, and the image of the borders of the inclusion weakens.

3.6. Comparison to SAFT. A quantitative comparison between RTM and SAFT imaging is not possible in the current stage. The decisive difference between them is the acquisition geometry. All the examples for RTM quoted above are calculated with a multioffset geometry. The available SAFT algorithms used for nondestructive testing require constant offset measurements. In addition the distance between shot points used for RTM is largely compared to SAFT. For a detailed SAFT imaging of concrete usually a distance of $0.02 \mathrm{~m}$ is used. For RTM we used $0.08 \mathrm{~m}$. However, we have been able to show that RTM is capable to image vertical and circular inhomogeneities, which is not possible with aperture limited approaches as SAFT. An example for results acquired by SAFT and RTM on a real concrete block was published by the same authors [14].

\section{Conclusion and Outlook}

By using different polyamide models and a concrete-like synthetic model we have demonstrated the potential of the RTM for nondestructive testing in civil engineering. Vertical borders and lower parts of built-in elements can be imaged clearly. The image quality can thus be improved, and more faults can probably be found compared to conventional imaging techniques.

Artefacts which have been seen in RTM images have to be carefully analysed and eliminated. For this task other imaging 


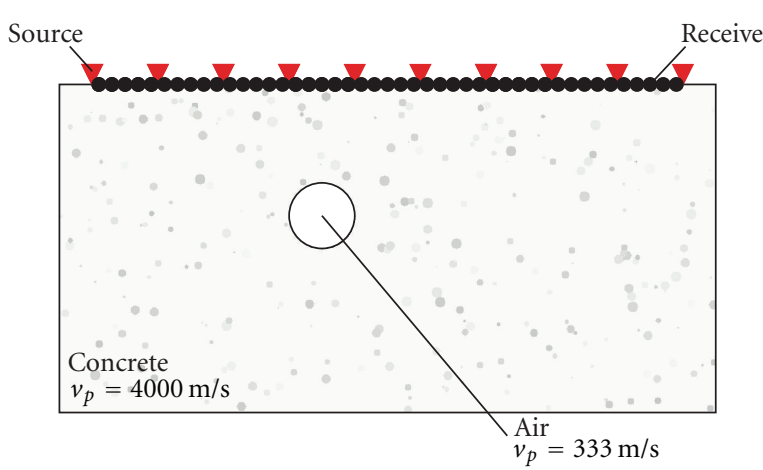

(a)

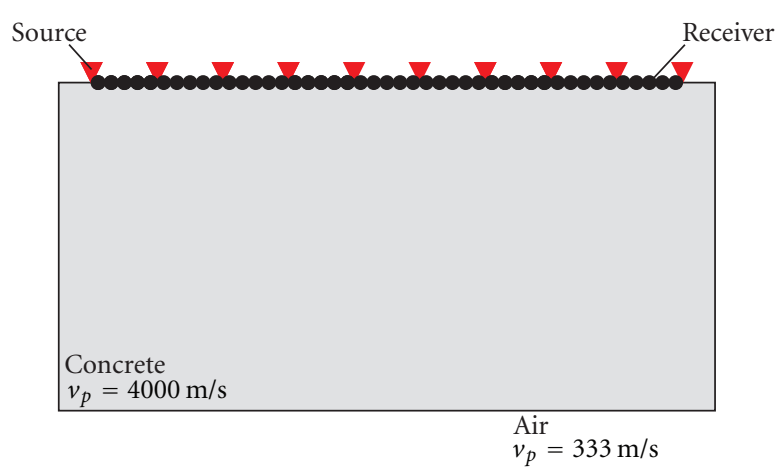

(b)

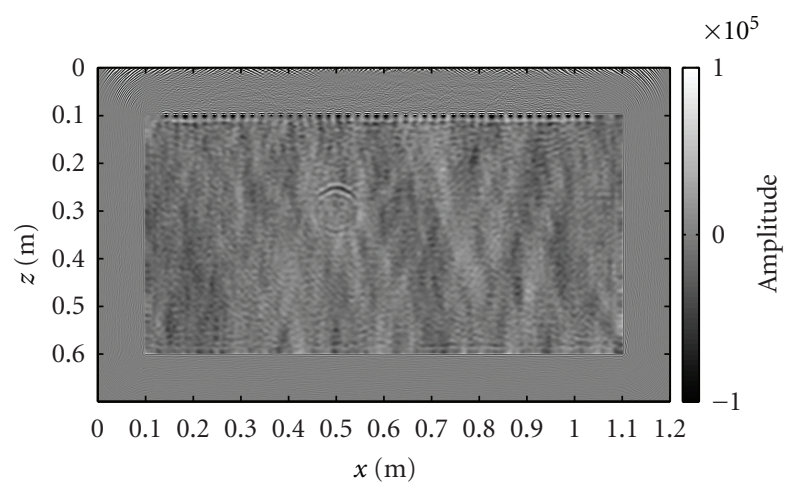

(c)

FIGURE 8: (a) Concrete-like synthetic model with an air inclusion. (b) Homogeneous velocity model for migration. (c) RTM imaging result using velocity model of $(b)$.

conditions than cross-correlation may be used but have not been explored by us so far. In addition, improved velocity models should be chosen for the RTM to improve the resulting image. For more complicated tasks a multistage RTM can be helpful to image all features. Experiments on test specimens of polyamide and concrete under laboratory conditions are currently performed to evaluate the synthetic results. Measurement equipment as well as acquisition parameters has to be tested and optimised to obtain a good signalto-noise ratio. The RTM code so far assumes point sources, while many sensors used in practice have a contact area with a size greater than wavelength.

Another topic to be worked on is to expand the algorithm to three dimensions and to use the full elastic wave equation. In nondestructive testing on concrete most measurements are carried out using shear waves, as smaller defects can be detected this way. To include this, the imaging conditions have to be adapted and the computing efficiency has to be improved as well. This can be done by keeping not every time step of the wave field calculation in RAM.

\section{Acknowledgments}

Wolfgang Baumann of the Zuse Institute in Berlin helped to implement the RTM code on their supercomputer. Special thanks to the author's colleagues at BAM 8.2, especially
Martin Krause and Christian Köpp, who helped in the preparation of the text. The remarks of two anonymous reviewers helped to improve the paper. All this is deeply appreciated.

\section{References}

[1] K. Mayer, R. Marklein, K. J. Langenberg, and T. Kreutter, "Three-dimensional imaging system based on fourier transform synthetic aperture focusing technique," Ultrasonics, vol. 28, no. 4, pp. 241-255, 1990.

[2] M. Schickert, M. Krause, and W. Müller, "Ultrasonic imaging of concrete elements using reconstruction by synthetic aperture focusing technique," Journal of Materials in Civil Engineering, vol. 15, no. 3, pp. 235-246, 2003.

[3] M. Krause, K. Mayer, M. Friese, B. Milmann, F. Mielentz, and G. Ballier, "Progress in ultrasonic tendon duct imaging," in Proceedings of the 7th International Symposium on Non Destructive Testing in Civil Engineering (NDTCE '09), Nantes, France, June 2009.

[4] M. Krause, B. Milmann, F. Mielentz et al., "Ultrasonic imaging methods for investigation of post-tensioned concrete structures: a study of interfaces at artificial grouting faults and its verification," Journal of Nondestructive Evaluation, vol. 27, no. 1-3, pp. 67-82, 2008.

[5] E. Baysal, D. D. Kosloff, and W. C. Sherwood, "Reverse time migration," Geophysics, vol. 48, no. 11, pp. 1514-1524, 1983. 
[6] D. Loewenthal and I. R. Mufti, "Reversed time migration in spatial frequency domain," Geophysics, vol. 48, no. 5, pp. 627635, 1983.

[7] G. A. McMechan, "Migration by extrapolation of time-dependent boundary values," Geophysical Prospecting, vol. 31, no. 3, pp. 413-420, 1983.

[8] P. A. Farmer, I. F. Jones, H. Zhou, R. I. Bloor, and M. C. Goodwin, "Application of reverse time migration to complex imaging problems," First Break, vol. 24, no. 9, pp. 65-73, 2006.

[9] L. Zhou, F. G. Yuan, and W. J. Meng, "A pre-stack migration method for damage identification in composite structures," Smart Structures and Systems, vol. 3, no. 4, pp. 439-454, 2007.

[10] L. Wang and F. G. Yuan, "Damage identification in a composite plate using prestack reverse-time migration technique," Structural Health Monitoring, vol. 4, no. 3, pp. 195-211, 2005.

[11] T. Bohlen, "Parallel 3-D viscoelastic finite difference seismic modelling," Computers and Geosciences, vol. 28, no. 8, pp. 887$899,2002$.

[12] A. Kurzmann, D. Koehn, A. Przebindowska, N. Nguyen, and T. Bohlen, "Acoustic full waveform tomography: performance and optimization," in Proceedings of the 70th EAGE Conference and Technical Exhibition, Amsterdam, The Netherlands, June 2009.

[13] B. Kaelin and A. Guitton, "Imaging condition for reverse time migration," SEG Technical Program Expanded Abstracts, vol. 25, no. 1, pp. 2594-2598, 2006.

[14] S. Müller, E. Niederleithinger, M. Krause, and T. Bohlen, "Reverse time migration: a seismic imaging technique applied to ultrasonic data," in Proceedings of the SMT Conference of NDE/NDT for Highways and Bridges: Structural Materials Technology (SMT), New York, NY, USA, August 2010. 

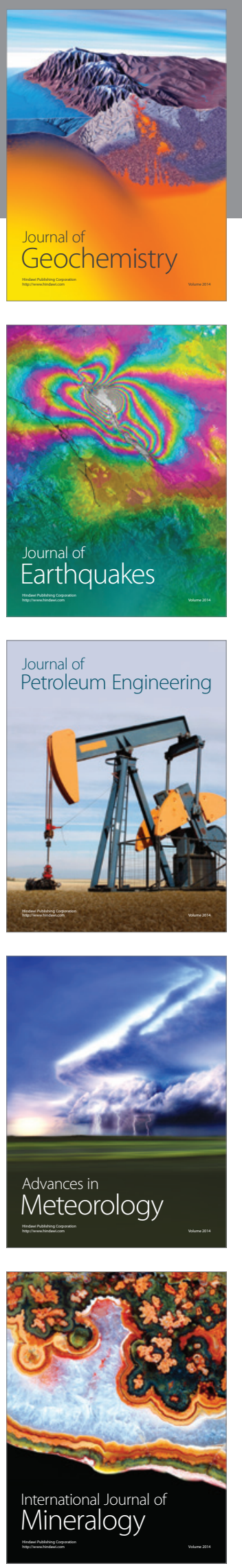
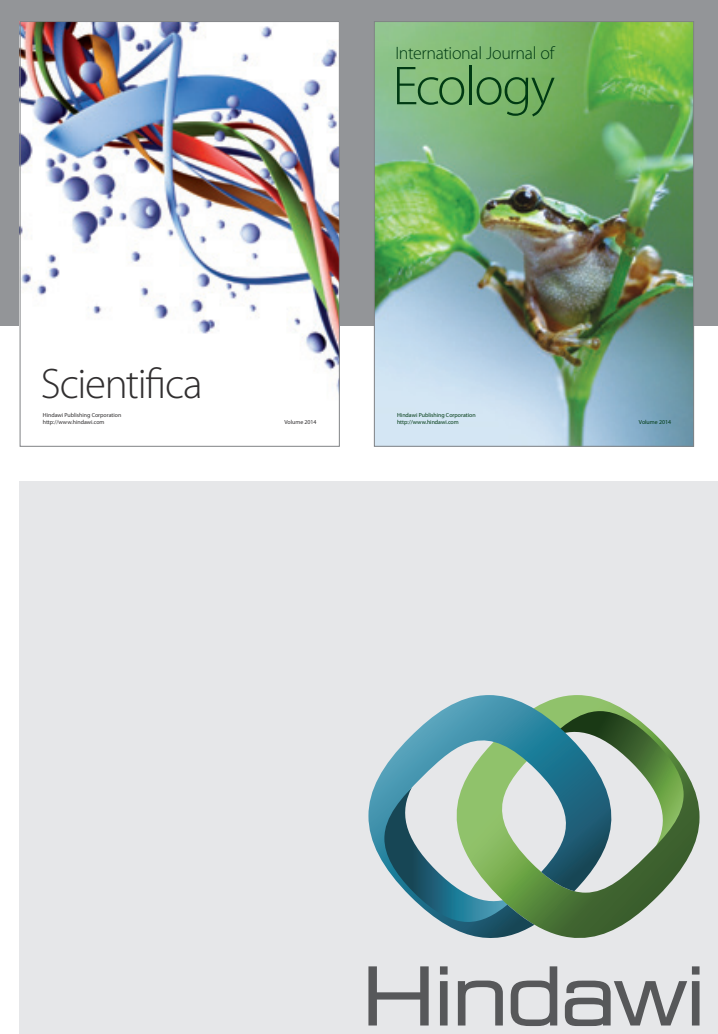

Submit your manuscripts at http://www.hindawi.com
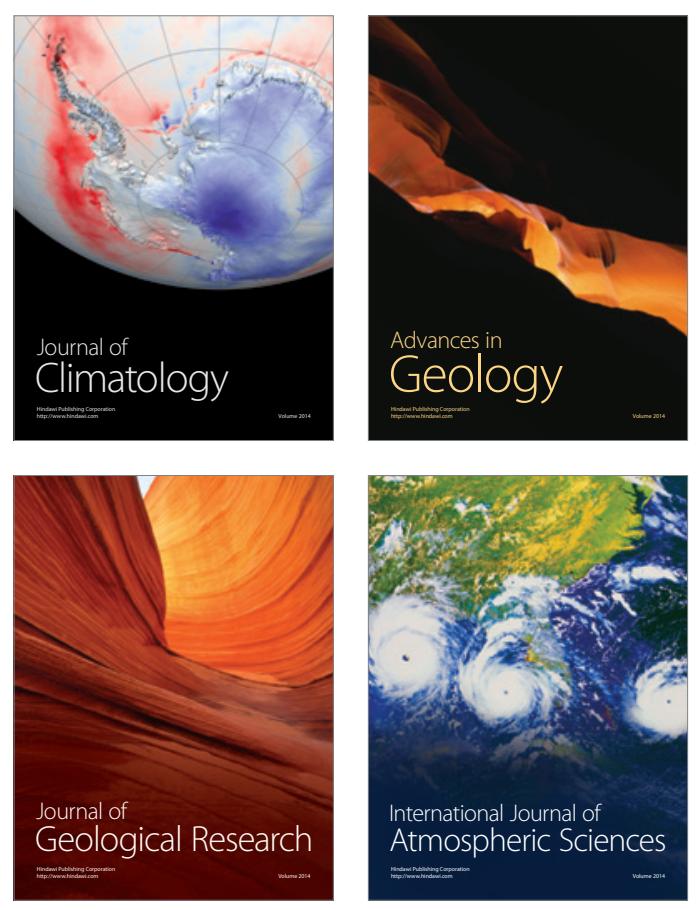
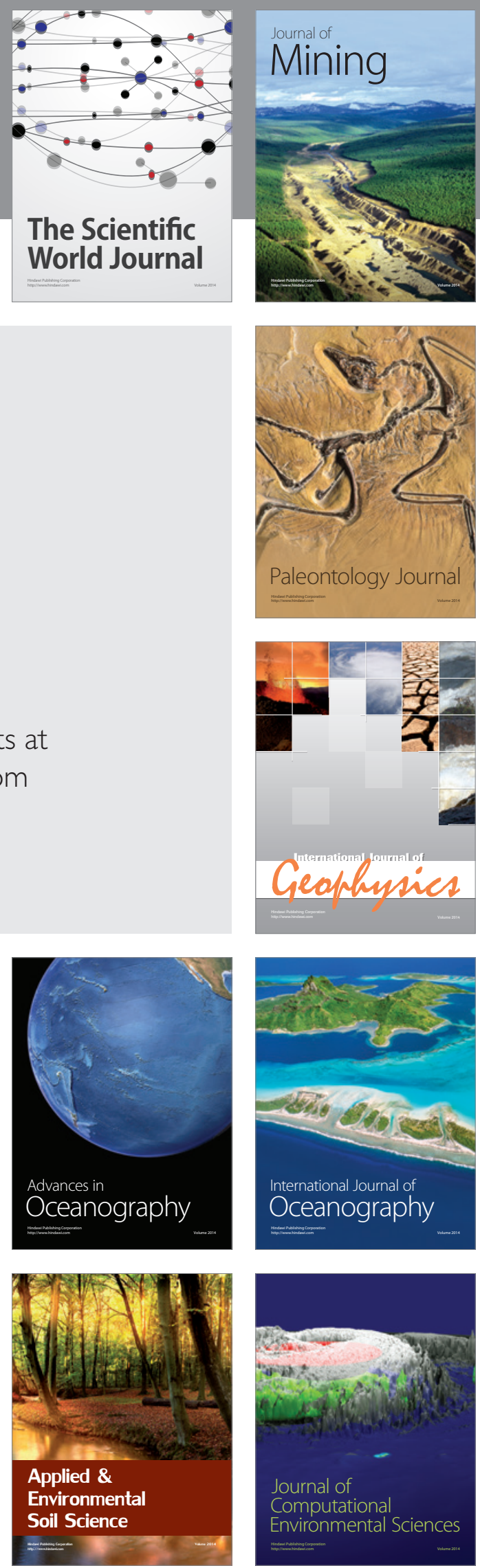